\title{
Parameter Identification and Control Scheme for Monitoring Automatic Thickness Control System with Measurement Delay
}

\author{
Xu Yang, ${ }^{1}$ Jingjing Gao, ${ }^{1}$ Yuri A. W. Shardt, ${ }^{2}$ Linlin Li, ${ }^{1}$ and Chaonan Tong ${ }^{1}$ \\ ${ }^{1}$ School of Automation and Electrical Engineering, University of Science and Technology Beijing, Beijing, China \\ ${ }^{2}$ Department of Chemical Engineering, University of Waterloo, Waterloo, ON, Canada \\ Correspondence should be addressed to Xu Yang; yangxu@ustb.edu.cn
}

Received 8 March 2017; Revised 24 April 2017; Accepted 10 May 2017; Published 21 June 2017

Academic Editor: Abdul-Qayyum Khan

Copyright (c) $2017 \mathrm{Xu}$ Yang et al. This is an open access article distributed under the Creative Commons Attribution License, which permits unrestricted use, distribution, and reproduction in any medium, provided the original work is properly cited.

\begin{abstract}
The thickness of the steel strip is an important indicator of the overall strip quality. Deviations in thickness are primarily controlled using the automatic gauge control (AGC) system of each rolling stand. At the last stand, the monitoring AGC system is usually used, where the deviations in thickness can be directly measured by the X-ray thickness gauge device and used as the input to the AGC system. However, due to the physical distance between the thickness detection device and the rolling stand, time delay is unavoidably present in the thickness control loop, which can affect control performance and lead to system oscillations. Furthermore, the parameters of the system can change due to perturbations from external disturbances. Therefore, this paper proposes an identification and control scheme for monitoring AGC system that can handle time delay and parameter uncertainty. The cross-correlation function is used to estimate the time delay of the system, while the system parameters are identified using a recursive least squares method. The time delay and parameter estimates are then further refined using the Levenberg-Marquardt algorithm, so as to provide the most accurate parameter estimates for the complete system. Simulation results show that, compared with the standard Proportion Integration Differentiation (PID) controller approach, the proposed approach is not affected by changes in the time delay and parameter uncertainties.
\end{abstract}

\section{Introduction}

The ever-increasing demand for product quality is the key issue for today's complex industrial processes. As a complex, large-scale process, the hot strip mill process is a typical example, where it is necessary to maintain deviations in strip thickness to within an acceptable range, using automatic control loops and subsystems. The final strip thickness is the most important variable to consider in the thickness control subsystem. The monitoring automatic gauge control (AGC) approach is an efficient way to implement control for such loops. However, difficulties with this approach include the inevitable time delay [1] due to the physical distance between the thickness detection device and the process, as well as system uncertainties caused by changes in operating conditions or external disturbances. These issues will lead to control performance degradation, which can lead to oscillations in the overall process [2]. In order to ensure product quality and guarantee that the rolling process is sustainable, reliable, and stable, it is important to monitor the complete rolling process in real time, especially for conditions that may affect product quality and equipment safety $[3,4]$. Therefore, in order to improve the performance, it is necessary to accurately identify the time delay and parameter uncertainties in the monitoring AGC system.

Time-delay estimation is an area of active research that seeks to develop efficient and accurate methods for estimating time delay from industrial data. Time-delay estimation can be divided into four broad categories [5]: time-delay approximation methods, explicit time-delay parameter methods, area and moment methods, and higher-order statistics methods. Of these methods, the area and moment methods are most commonly used for industrial data. A common area and moment method is the cross-correlation function, which can be used to estimate the delay between two signals [6]. The accuracy and speed of this approach make it very 
suitable for automated industrial implementation. Other approaches include fitting estimated models to the data set to backcalculate the optimal time delay [5, 7], using MATLAB's delay function to compute the values, using support vector machines $[7,8]$, performing nonlinear optimization using the steepest descent method [9], and using step tests to determine the values.

Similarly, online, parameter estimation is a common area of research that focuses on determining the system parameters as the conditions of the system change. A common approach is to use a recursive method to estimate the parameters after every new sample of information arrives. Such an approach has the advantage that it will track the process changes quickly. A common implementation of the recursive method is the recursive least squares approach, which uses a linear regression framework to update the parameter estimates [10-12]. Other methods include optimization methods using stochastic gradient descent methods [8], implementing a variable search regions $[13,14]$, and various neural network identification methods $[15,16]$.

Finally, various methods have been proposed to handle time delays and uncertain process information, including the Smith predictor [17-19], soft-sensor-based control [20, 21], and robust fault-tolerant control [22-24]. Of these methods, model-based control methods, such as the Smith predictor and internal model control, can effectively compensate for the influences of time delay, but they are not suitable for complex systems with parameter uncertainties.

For the rolling mill process, first-principle models are hard to determine precisely due to the complex nature and harsh operating conditions of the process. For this reason, model-based monitoring and control methods are hard to implement for such complex systems. However, for many complex industrial processes, process data is easy to obtain and PID-based control is still widely used. Therefore, it is advantageous to use data-driven identification [25] and control methods $[26,27]$ that can use the process data efficiently while maintaining, as much as possible, the existing control structure.

Therefore, the objectives of this paper are: to develop a framework for handling uncertainties in the process model; to investigate the use of the cross-correlation function and recursive least squares to obtain parameter estimates, which are then refined using nonlinear optimization, such as the Levenberg-Marquardt method; and to test the proposed results using a simulated hot steel rolling mill process.

\section{Monitoring AGC System Analysis}

2.1. General Description of AGC System. In hot/cold tandem rolling mills, in terms of product quality, the key unit is the finishing mill group, which consists of 5 mill stands in cold rolling and 7 mill stands in hot rolling. The strip passes through a roll gap in each stand and comes in contact with a pair of work rolls that are driven by an electric motor from the main drive system and are supported by a pair of backup rolls with a larger diameter. As shown in Figure 1, there are two hydraulic reduction devices on each side of the top backup

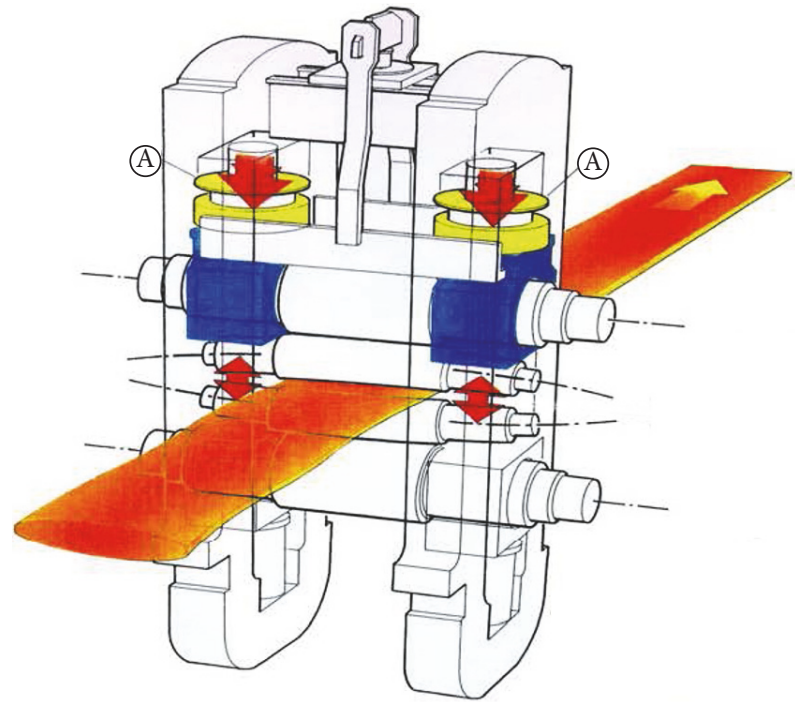

(A) HAGC cylinder

FIGURE 1: The mechanical structure of finishing mill stand.

roll, which indirectly screw down onto the rolled strip via force transmission between the backup and work rolls.

The hydraulic reduction device is a strip gauge control system that includes the mechanical parts such as servo amplifier, servo-valve, hydraulic cylinder and electrical parts, such as a digital controller and sensors. Thus, it can be defined as a typical electromechanical-hydraulic coupling system [28], also known as the automatic gauge control (AGC) system. There are several control methods for the AGC system, which can be divided into direct/indirect thickness measurement control structures, which mainly depend on whether the thickness deviation can be obtained by X-ray thickness sensors or model calculations.

The monitoring AGC system is usually used at the last rolling stand, where the thickness deviation can be directly measured by the X-ray thickness gauge device and then can be used as system output in thickness control loop. However, due to the physical distance between the thickness detection device and the rolling stand, a measurable time delay is unavoidable, which can result in control performance degradation that in extreme cases can lead to control system instability.

2.2. Mathematical Model of the Monitoring AGC System. Figure 2 shows the structure of the monitoring AGC system, which consists of two control loops: a Hydraulic Automatic Position Control (HAPC) inner loop and an X-ray thickness monitoring outer loop. Figure 3 shows a block diagram of the monitoring AGC system.

As shown in Figure 3, let the expected strip exit thickness be $h_{0}$ and the actual exist thickness measured by the X-ray thickness gauge be $h$. Thus, the thickness error $\Delta h$ defined as $h_{0}$ minus $h$ is fed back to the monitoring AGC system to adjust the roll gap to eliminate the thickness error. The output from the PID controller is fed into the Hydraulic Automatic Position Control (HAPC) system. 


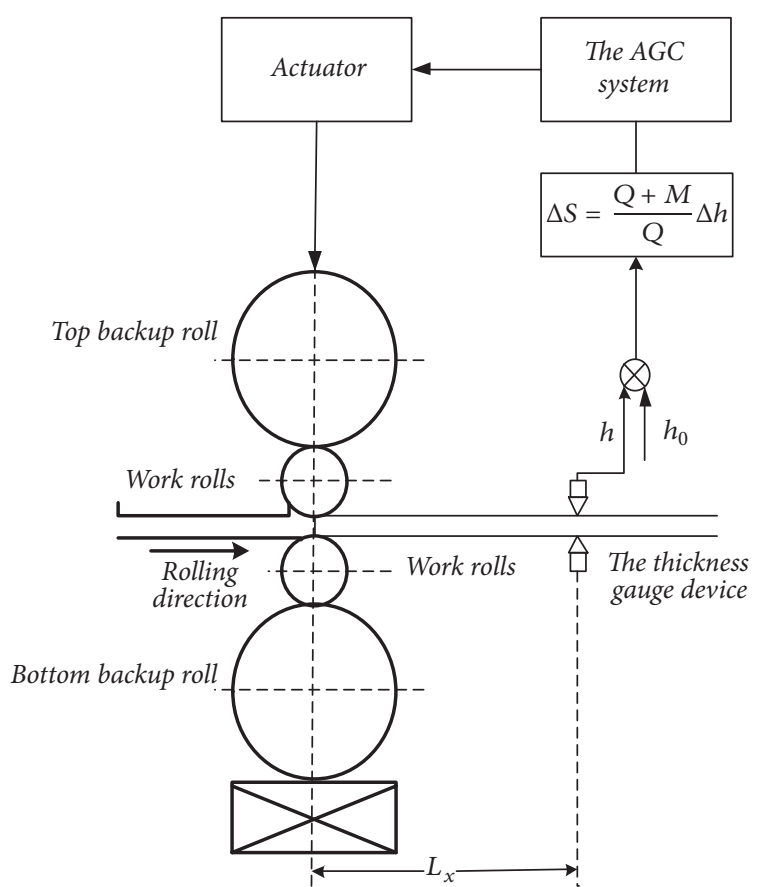

FIGURE 2: The structure of the monitoring AGC system.

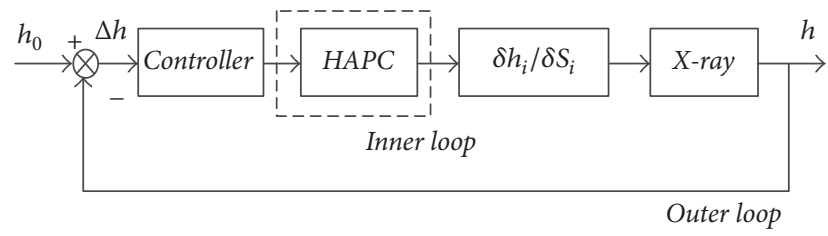

FIGURE 3: Block diagram of the monitoring AGC system.

Since the AGC and HAPC systems contain many mechanical and electrical components that interact in a complex manner, the resulting model can be quite complex. However, since the inertia of most of the components is very small, the transfer function of the HAPC closed-loop system can be described by a simple first-order plus dead time (FOPDT) model:

$$
\frac{\delta S}{\delta S_{R}}=\frac{1}{\tau_{1} s+1}
$$

where $\delta S_{R}$ is the adjustment of the roll gap set point value and $\tau_{1}$ is the time constant of the HAPC system. Based on the actual system, the relationship between the thickness and the roll gap can be approximated as a FOPDT model [29]

$$
\frac{\delta h}{\delta S}=\frac{M}{M+Q} \times \frac{e^{-\theta s}}{\tau_{2} s+1},
$$

where $\delta h$ represents the exit thickness, $M$ is the mill stand stiffness, $Q$ is the plastic stiffness coefficient of the strip, $\tau_{2}$ is the time constant, and $\theta$ is the time delay in the monitoring AGC system. This time delay is a result of the distance between the X-ray thickness gauge device and the last stand and can be defined as

$$
\theta=\frac{L_{x}}{v_{m}}
$$

where $L_{x}$ is the distance between the X-ray thickness gauge device and the last stand and $v_{m}$ is the exit speed of the steel at the last stand.

Using block diagram algebra, the transfer function for the overall process can be written as

$$
\frac{y(s)}{u(s)}=\frac{\delta h}{\delta S_{R}}=\frac{\delta S}{\delta S_{R}} \frac{\delta h}{\delta S}=K \frac{e^{-\theta s}}{\left(\tau_{1} s+1\right)\left(\tau_{2} s+1\right)},
$$

where $K$ is the overall gain of the process, $y$ is the exit thickness of the steel, and $u$ is the roll gap adjustment set point.

The process described by transfer function (4) is the ideal process without any perturbations due to external disturbances or internal uncertainties. Also, the time delay can vary due to changes in steel characteristics. Therefore, during the course of operation, it is important to monitor the process and determine if the parameters have changed their value. If so, it is necessary to update the values of the model parameters in order to minimize plant-model mismatch.

\section{Parameter Identification and Control Algorithm}

In order to perform both identification and control of the process within the same structure, a novel 4-step approach is used. Figure 4 shows a block diagram of the key components of this approach. The 4 main steps are as follows:

(1) Time Delay Estimation. Using the available offline input and output data, compute the time delay using the cross-correlation method.

(2) Parameter Identification I (Recursive Least Square (RLS) Method). Using the recursive least squares method, identify the process parameters given the time delay from Step (1).

(3) Parameter Identification II (Levenberg-Marquardt Algorithm). Using the Levenberg-Marquardt (LM) algorithm, obtain the refined model parameters and time delay. The residual, that is, the difference between the predicted output, $y_{m}(k)$, and the actual online output, $y(k)$, is used as the objective function in order to obtain a better estimate of the model parameters, including the time delay.

(4) Control Action Determination. Calculate the final predicted model value, $\hat{y}(k)$, using the best parameter estimates from (3). Feed back this value to the controller to implement the next control step.

Thus, without changing the existing PID controller, the impact of time delay and parameter uncertainties on the monitoring AGC system is resolved and better closed-loop control is achieved. 


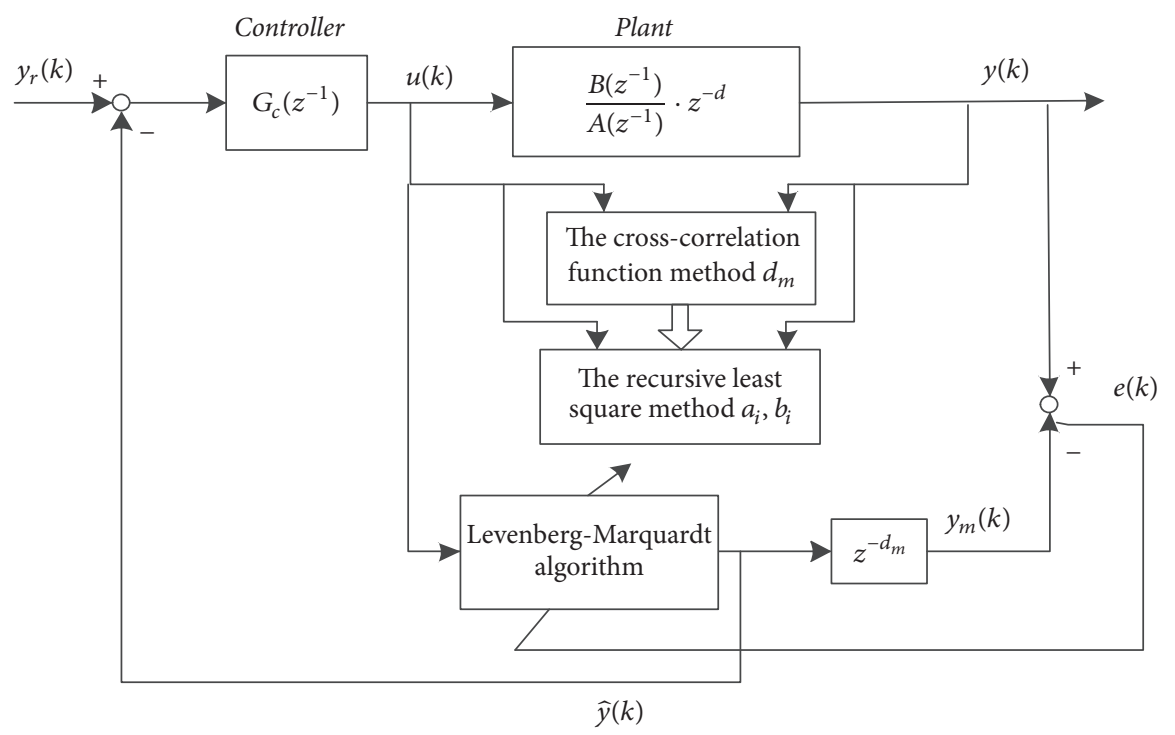

FIGURE 4: An identification and control scheme for the monitoring AGC system.

3.1. Process Model. In order to obtain a model that can easily be used for recursive least squares analysis, the model given by (4) will be discretized assuming a 1-second sampling rate. Also, it will be assumed that an autoregressive error term driven by Gaussian, white noise will be added to the process to model the disturbances. The autoregressive component of the model will have the same form as the denominator of the process model; that is, the final model will be an autoregressive model with exogenous input (ARX) [25], which can be written as

$$
A\left(z^{-1}\right) y(k)=z^{-d} B\left(z^{-1}\right) u(k)+\varepsilon(k),
$$

where $y(k)$ is the process output, $u(k)$ is the process input, $\varepsilon(k)$ is a Gaussian white noise disturbance, and

$$
\begin{aligned}
& A\left(z^{-1}\right)=1+a_{1} z^{-1}+a_{2} z^{-2} \quad\left(a_{0}=1\right) \\
& B\left(z^{-1}\right)=b_{1} z^{-1}+b_{2} z^{-2} \quad\left(b_{0}=0\right) .
\end{aligned}
$$

$a_{1}, a_{2}, b_{1}$, and $b_{2}$ are the parameters to be identified and $d$ is the discrete time delay. It should be noted that the discrete and continuous time delays are related by

$$
d=\left\lfloor\frac{\theta}{\tau_{s}}\right\rfloor,
$$

where $\tau_{s}$ is the sampling rate and $\lfloor\cdot\rfloor$ is the round-down function.

It should be noted that ARX models are one of the few classes of discrete models that can be directly solved using linear regression [30]. This implies that it will be possible to use recursive least squares to estimate the parameter estimates.

3.2. Time-Delay Estimation Using the Cross-Correlation Method. The cross-correlation method can be used to obtain the delay parameter accurately $[7,25]$. In this method, the cross-correlation between the input and output signals is computed for different lags between the signals in order to determine the time delay.

Given two signals $u(k)$ and $y(k)$, the cross-correlation between $u$ and $y$ at a lag of $\tau$, denoted by $R_{u y}(\tau)$, is defined as [25]

$$
R_{u y}(\tau)=\frac{1}{N} \sum_{k=1}^{N} u(k) y(k+\tau) .
$$

In order to determine the best value, $\tau$ will be computed for a series of values ranging from 1 to $n$, where $n \lll$ $N$. The first nonzero lag will correspond to the time delay. In order to improve the accuracy of the computation, the cross-correlation between the signals will be computed in the Fourier (or frequency) domain [31]. Furthermore, it can be noted that using the frequency domain for determining the time delay is common in signal processing. Algorithm 1 gives the procedure used for estimating the time delay.

Algorithm 1 (algorithm for estimating the time delay using the cross-correlation method).

Step 0 . Get the offline input, $u(k)$, and output, $y(k)$, data.

Step 1. Compute the discrete Fourier transform of $u(k)$ and $y(k)$.

Step 2. Calculate the cross-correlation function between the transformed signals as a function of different lags.

Step 3. Compute the inverse discrete Fourier transform of the cross-correlation function and find the discrete time point $k$ corresponding to its maximum. The estimated time delay, $\widehat{\theta}$, would then be

$$
\widehat{\theta}=\left|k-\frac{N}{2}+1\right| \tau_{s}
$$


3.3. Parameter Estimation I: Recursive Least Square (RLS) Method. Since in the monitoring AGC system, sensors and the thickness gauge device can provide real-time process information, it is possible to use the recursive least square (RLS) method to update the system parameters.

In order to use the RLS method, it is first necessary to cast the model given by (5) into the standard linear regression problem [25]:

$$
y(k)=\varphi^{T}(k) \beta(k)+\varepsilon(k),
$$

where the vector, $\varphi^{T}(k)$, is defined as

$$
\begin{aligned}
& \varphi^{T}(k) \\
& =\left[\begin{array}{rr}
y(k-1), y(k-2), \ldots & \\
& u(k-1-d), u(k-2-d)
\end{array}\right],
\end{aligned}
$$

the parameter vector, $\beta(k)$, is defined as

$$
\beta(k)=\left[a_{1}, a_{2}, b_{1}, b_{2}\right]^{T},
$$

and $\varepsilon(k)$ is a Gaussian signal.

The RLS method can be written as [10]

$$
\widehat{\beta}(k)=\widehat{\beta}(k-1)+K(k)\left[y(k)-\varphi^{T}(k) \widehat{\beta}(k-1)\right],
$$

where $K(k)$ is the gain computed using

$$
K(k)=\frac{P(k-1) \varphi(k)}{\lambda+\varphi^{T}(k) P(k-1) \varphi(k)} .
$$

$\lambda$ is the forgetting factor and $P(k)$ is the inverse of the regress or matrix, which can be recursively computed using

$$
P(k)=\lambda^{-1}\left[I-K(k) \varphi^{T}(k)\right] P(k-1)
$$

The forgetting factor, $\lambda$, represents the weight assigned to previous errors. The smaller the value, the greater the influence of the current values on the final estimate. In the proposed approach, $\lambda=1$ since it is desired to implement a standard, linear regression algorithm without any weighting for past values; that is, all past errors are to be considered equally.

Algorithm 2 summarizes the steps for implementing the RLS method in the proposed framework.

Algorithm 2 (estimating the process parameters of the monitoring AGC system using the recursive least square method).

Step 0 . Set the initial values for $\widehat{\beta}(0)$ and $P(0)$.

Step 1. Get the offline data for input, $u(k)$, and output, $y(k)$.

Step 2. Compute $\widehat{\beta}(k)$ using (13) and update $K(k)$ and $P(k)$ using (14) and (15).

Step 3. Increase $k$ by 1 , and go to Step 1 until $\widehat{\beta}(k)$ has converged.
3.4. Parameter Estimation II: Levenberg-Marquardt (LM) Algorithm. The Levenberg-Marquardt (LM) algorithm is the most widely used nonlinear least squares algorithm, which combines the advantages of both the gradient descent and the Gauss-Newton methods.

Consider the general least squares minimization problem that can be formulated as

$$
\min f(\vec{x})=0.5 \sum_{k} r_{k}^{2}(\vec{x}),
$$

where $r$ is the residual and $x$ is a vector of size $n$. Let the residual vector, $r(x)$, be defined as

$$
\vec{r}(\vec{x})=\left(r_{1}(\vec{x}), r_{2}(\vec{x}), \ldots, r_{m}(\vec{x})\right)
$$

then the objective function, $f(x)$, can be rewritten as

$$
f(\vec{x})=0.5\|\vec{r}(\vec{x})\|^{2} .
$$

Let the Jacobian matrix, $J$, of $r$ with respect to $x$ be defined as

$$
J_{j i}=\frac{\partial r_{j}}{\partial x_{i}}
$$

where $j=1,2, \ldots, m$ and $i=1,2, \ldots, n$. It follows that the gradient of $f(x)$, denoted by $\nabla f$, can be written as

$$
\nabla f(\vec{x})=\sum_{j=1}^{m} r_{j}(\vec{x}) \nabla r_{j}(\vec{x})-J(\vec{x})^{T} \vec{r}(\vec{x})
$$

and the Hessian, denoted by $\nabla^{2} f$, is given as

$$
\nabla^{2} f(\vec{x})=J(\vec{x})^{T} J(\vec{x})+\sum_{j=1}^{m} r_{j}(\vec{x}) \nabla^{2} r_{j}(\vec{x}) .
$$

When the residuals are small or the gradient of $r_{j}$ is small, then the second term in (21) reduces to zero and the Hessian can be written as simply

$$
\nabla^{2} f(\vec{x})=J(\vec{x})^{T} J(\vec{x}) .
$$

In order to obtain a solution for the optimization problem, let us consider a second-order Taylor series expansion of $\nabla f$ around $x_{0}$. That is,

$$
\nabla f(\vec{x})=\nabla f\left(\vec{x}_{0}\right)+\left(\vec{x}-\vec{x}_{0}\right) \nabla^{2} f\left(\vec{x}_{0}\right) .
$$

Setting (21) to zero yields

$$
\vec{x}_{i+1}-\vec{x}_{i}=\left(\nabla^{2} f\left(\vec{x}_{i}\right)\right)^{-1} \nabla f\left(\vec{x}_{i}\right) .
$$

After substitution of (22) into (24), then Hessian matrix can be solved. However, such a naïve implementation can encounter numerical stability problems. Therefore, the Levenberg-Marquardt algorithm replaces the Hessian by

$$
J^{T} J+\lambda \operatorname{diag}\left(J^{T} J\right)
$$


TABLE 1: Parameters of the monitoring AGC system.

\begin{tabular}{lcc}
\hline Parameter & Value & Descriptions \\
\hline$M$ & $5,800 \mathrm{kN} / \mathrm{mm}$ & Mill stand stiffness \\
$Q$ & $31,006 \mathrm{kN} / \mathrm{mm}$ & Plastic stiffness coefficient of strip \\
$\tau_{1}$ & $0.01 \mathrm{~s}$ & Time constant of the HAPC system \\
$\tau_{2}$ & $0.5 \mathrm{~s}$ & Time constant of the first-order inertia \\
$L_{m}$ & $6 \mathrm{~m}$ & Distance between the X-ray thickness gauge device and the last stand \\
$v_{m}$ & $12 \mathrm{~m} / \mathrm{s}$ & Exit speed of the steel at the last stand \\
$h_{0}$ & $2.0 \mathrm{~mm}$ & Thickness set point \\
$\tau_{s}$ & $0.1 \mathrm{~s}$ & Sampling time \\
\hline
\end{tabular}

where diag is the function that takes the diagonal entries of the matrix and $\lambda$ is a tuning parameter. Thus, (24) becomes

$$
\begin{aligned}
\vec{x}_{i+1} & -\vec{x}_{i} \\
= & \left(J^{T}(\vec{x}) J(\vec{x})+\lambda \operatorname{diag}\left(J^{T}(\vec{x}) J(\vec{x})\right)\right)^{-1} \nabla f\left(\vec{x}_{i}\right) .
\end{aligned}
$$

In the Levenberg-Marquardt algorithm, the tuning parameter is automatically updated based on the size of the current and previous errors. If the error increases, increase the value of $\lambda$ by $\gamma$ and try again. If the error decreases, decrease the value of $\lambda$ by $\gamma$ and keep the current values and perform a new iteration. New iterations are computed until the error stops decreasing or some relative tolerance, $\varepsilon$, has been reached.

In the problem at hand, it is desired to obtain optimal parameter and time-delay values by minimizing the residuals of the process; that is,

$$
\begin{aligned}
& J(\beta, k)=\sum e^{2}(\beta, k) \\
& e(\beta, k)=y(k)-\widehat{y}_{m}(\beta, k),
\end{aligned}
$$

where $e(\beta, k)$ is the residual between the predicted output, $\widehat{y}_{m}(\beta, k)$, and the actual output $y(k)$. The implementation of the LM algorithm for the proposed problem is shown as Algorithm 3.

Algorithm 3 (refining the process parameters and time delay of the monitoring AGC system using the Levenberg-Marquardt algorithm).

Step 0 . Set the initial values for the tolerance, $\varepsilon$, and the values of $\gamma$ and $\lambda$.

Step 1. Calculate the predicted output and the residual vector $r$, using the online values of $u(k)$ and $y(k)$.

Step 2. Calculate the Jacobian matrix, J, using (19).

Step 3. Compute $x_{i+1}-x_{i}$ using (26). Compute the current value of the objective function using (27).

Step 4. Check if $J\left(x^{(k)}\right)<\varepsilon$. If yes, stop the algorithm. If no, let $x^{(k+1)}$ be the new weight and threshold and calculate the target performance function as $J\left(x^{(k+1)}\right)=x^{(k+1)} J\left(x^{(k)}\right)$.

Step 5. Check if $J\left(x^{(k+1)}\right)<J\left(x^{(k)}\right)$. If yes, let $k=k+1$ and $\lambda=\lambda / \gamma$ and go to Step 1. If no, do not update the weights $x^{(k+1)}$, let $x^{(k+1)}=x^{(k)}$ and $\lambda=\gamma \lambda$, and go to Step 3 until $J\left(x^{(k)}\right)<\varepsilon$.

3.5. Control Action Implementation. Once the LM algorithm has optimized the residuals between the predicted output and the actual exit thickness to obtain the time delay and the system parameters which are close to the real system, the realtime estimated thickness without time delay can be obtained. This real-time estimated thickness can then be fed back to the controller.

This method greatly reduces the impact of time delay on the system. Moreover, the LM algorithm can identify the system parameters online, which results in better control performance for monitoring AGC system.

\section{Case Study}

To verify the feasibility and effectiveness of the proposed algorithm for handling parameter uncertainties and time delay in the monitoring AGC system, in this section, a case study is presented based on an actual finishing mill. All parameters, shown in Table 1, are based on actual industrial parameters for a finishing mill.

4.1. Time-Delay Estimation. Using the rolling process input/ output data, an initial estimate of the time delay can be obtained using the cross-correlation function method. Figure 5 shows the process data, while Figure 6 shows the crosscorrelation function for the given data.

As shown in Figure 6, the cross-correlation function reaches a maximum around 13,953 samples on a total length of 27,920 samples. The sampling time is $0.1 \mathrm{~s}$. Using (3), the time delay can be estimated as $0.5 \mathrm{~s}$. Note that using $L_{m}, v_{m}$, and (3) gives a similar result, suggesting that the time delay obtained using the cross-correlation method is accurate.

4.2. Initial Parameter Estimation Using RLS. Once the time delay has been preliminarily estimated using the cross-correlation method, an initial estimate of the parameters can be obtained using RLS. The initial value for $P, P(0)$, is set as $10^{6} I$ and $\theta(0)=[0,0,0,0]$. Figure 7 shows the estimated parameters using RLS as a function of time and their convergence speeds. As shown in Figure 7, the estimated parameters are $a_{1}=-0.8188, a_{2}=0.0000, b_{1}=0.0259$, and $b_{2}=$ 0.0026 . These values and the estimated time delay are then 


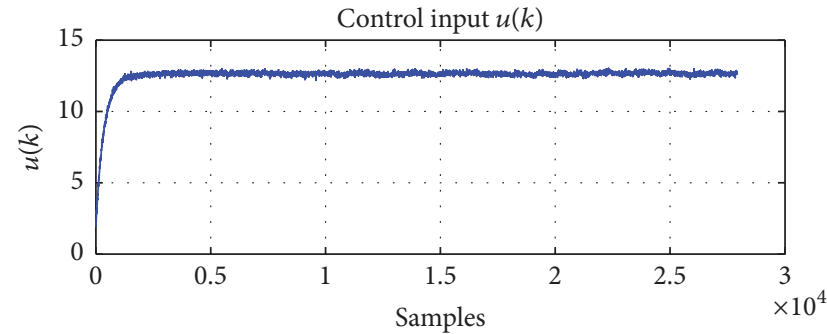

(a)

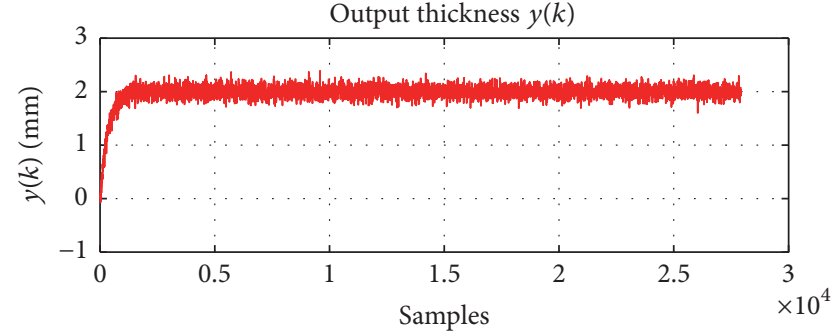

(b)

Figure 5: Process Data: (a) $u(k)$ and (b) $y(k)$.

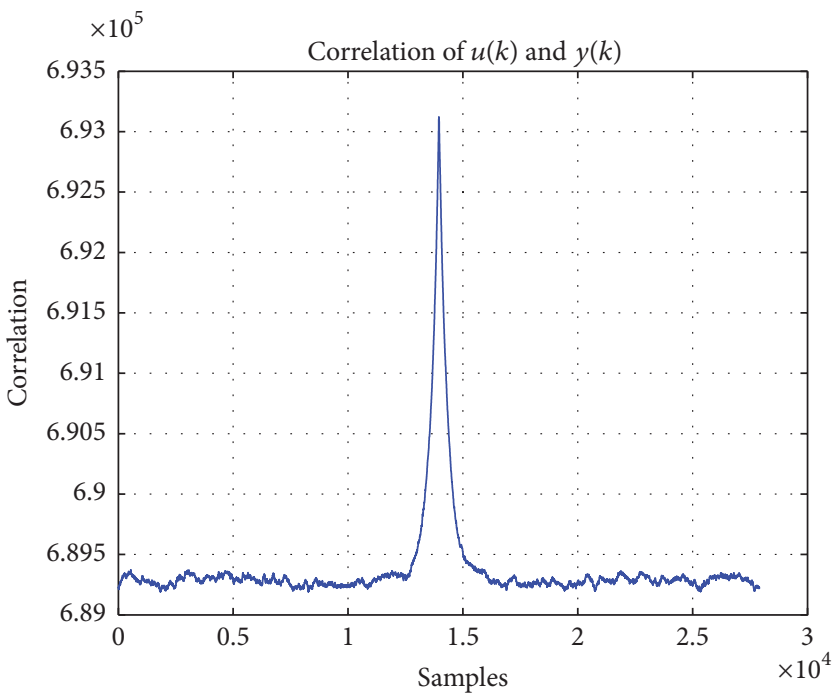

FIgURE 6: The cross-correlation function for the data set.

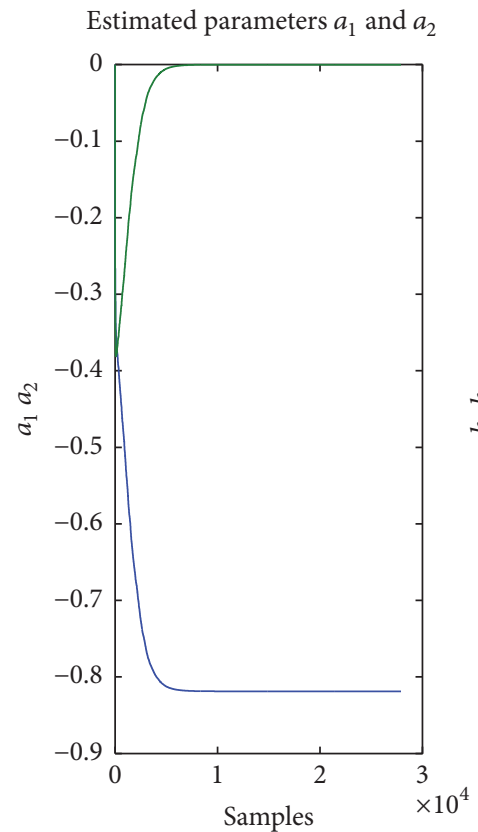

Estimated parameters $b_{1}$ and $b_{2}$

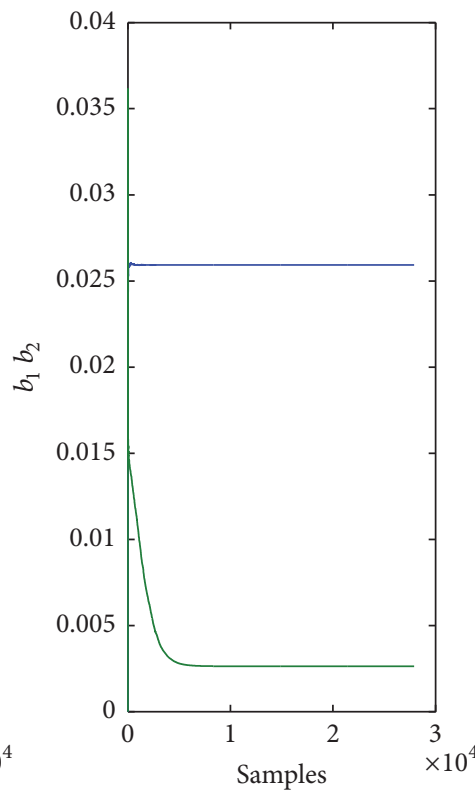

$\begin{array}{ll}-a_{1} & -b_{1} \\ -a_{2} & -b_{2}\end{array}$

FIGURE 7: Estimated process parameters using RLS. 

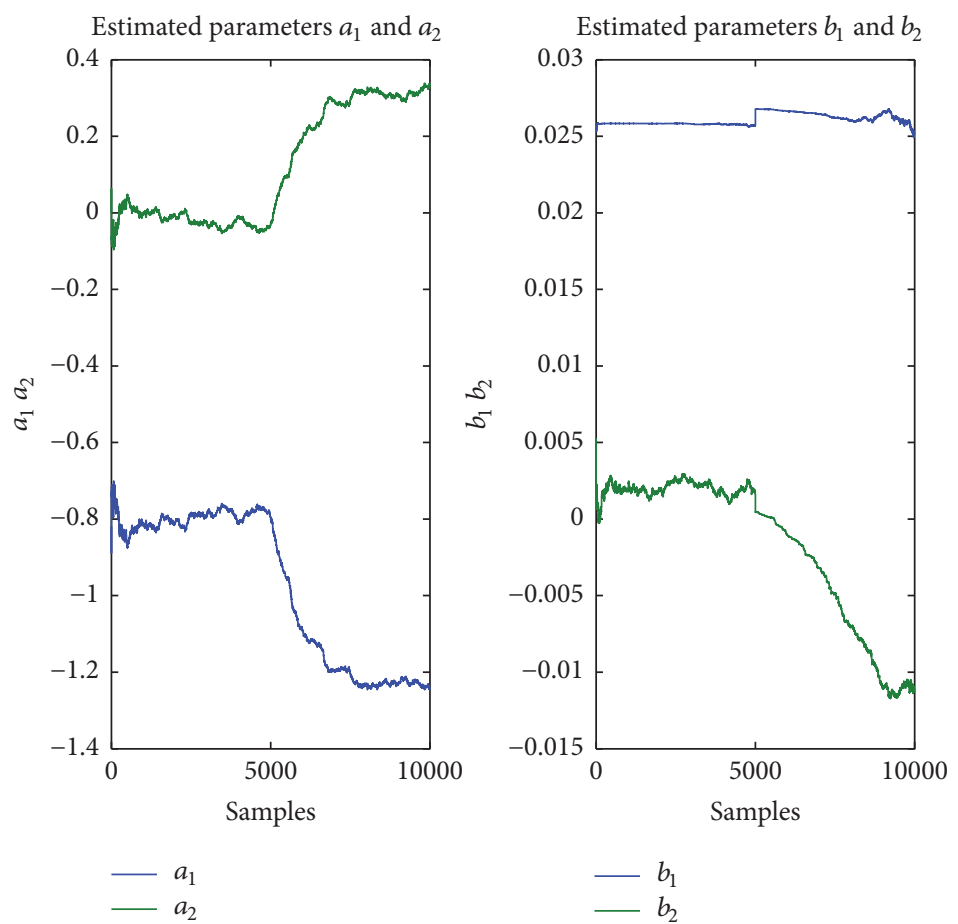

FIGURE 8: Refined process parameters using the LM algorithm.

used as the initial guess for the final parameter estimation stage using the LM algorithm.

4.3. Final Parameter Estimation. Since, in the actual process, the parameters of the system change, it is necessary to update the system using the LM algorithm to obtain accurate parameter estimates of the current system. For the purposes of this simulation, a disturbance signal is added at the 5,000th sample.

The parameter values are set as follows: threshold, $\varepsilon=0.1$, proportion coefficient, $\lambda=1$, and $\gamma=0.5$. The initial values of the time delay and parameter values are obtained using the cross-correlation method and RLS.

Figure 8 shows the evolution of the parameters as a function of sample time. Firstly, it can be noted that the parameter estimates converge to a given value. Secondly, it can be seen that the proposed method is able to update the process parameters as soon as the change has occurred. At the 10,000th sample, the new process values are $a_{1}=-1.2242$, $a_{2}=0.3128, b_{1}=0.0245$, and $b_{2}=-0.0105$.

Figures 9 and 10 show the comparison between the measured and estimated thickness for 10,000 samples. It is quite clear that the estimated thickness, $y_{m}(k)$, tracks well the actual output, $y(k)$, as shown in Figure 11. These figures all show the effectiveness of the proposed overall method.

4.4. Control Implementation. The final step is to consider the complete system and see its impact on the overall control structure.

Figures 12 and 13 show a comparison of the proposed system with the standard PID control. It can be seen that

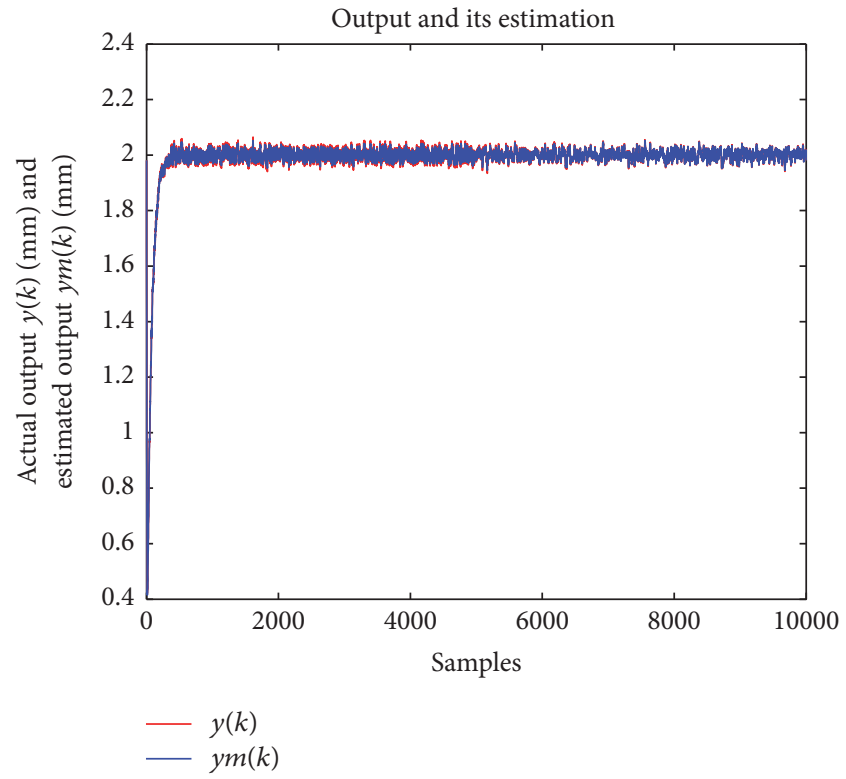

FIGURE 9: Measured and estimated output $y(k)$.

the PID control results experience more and larger deviations than the proposed system.

Since the process model changes at the 5,000th sample, it is convenient to consider the performance of the controllers before and after this point. For the PID controller, the variance of the output is 0.0274 before 5,000 and 0.0301 after. As expected, the variance has increased, since the controller is 


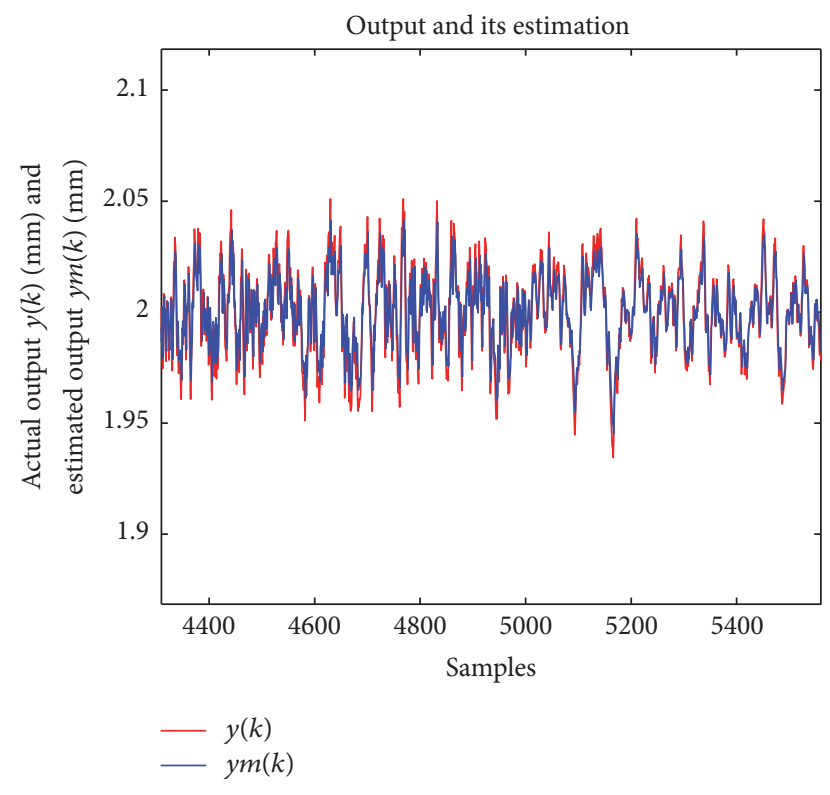

FIGURE 10: Detailed view of the true and estimated output $y(k)$.

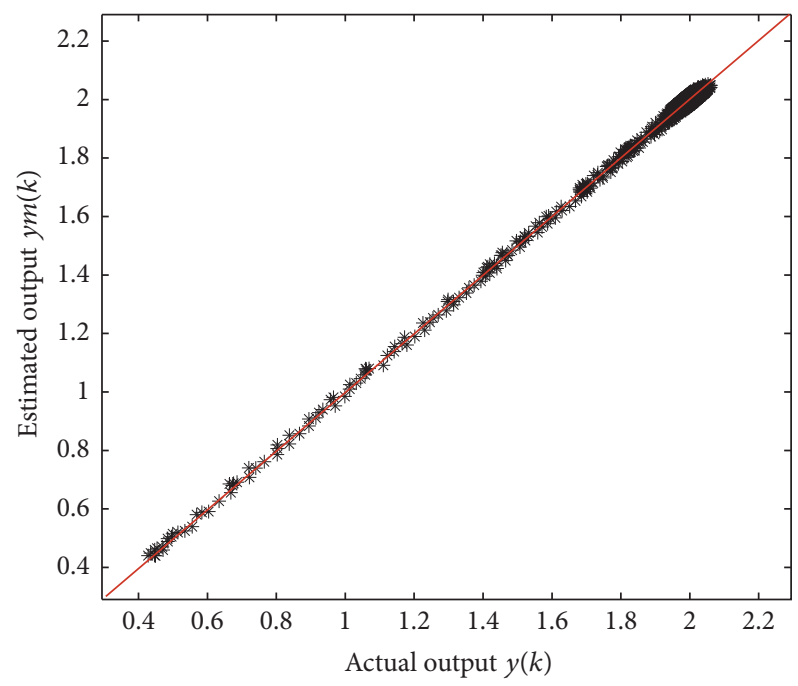

FIGURE 11: Comparison between $y(k)$ and its estimate, $y_{m}(k)$.

no longer properly designed given the changes in the overall system. This mismatch will lead to an increase in the output variance.

For the proposed new approach, the output variance before 5,000 is 0.0218 and 0.0165 after. Firstly, it can be noted that the variance in the first part is lower compared with the PID control loop, which implies that, even in the best design conditions, there still exists some plant-model mismatch that the new approach can identify to improve the overall performance. Furthermore, in the second part, the performance of the proposed new approach is not only lower than in the first case but also much smaller than the PID variance. This shows the key strength of the proposed new approach that it can effectively handle new conditions without requiring any external intervention.

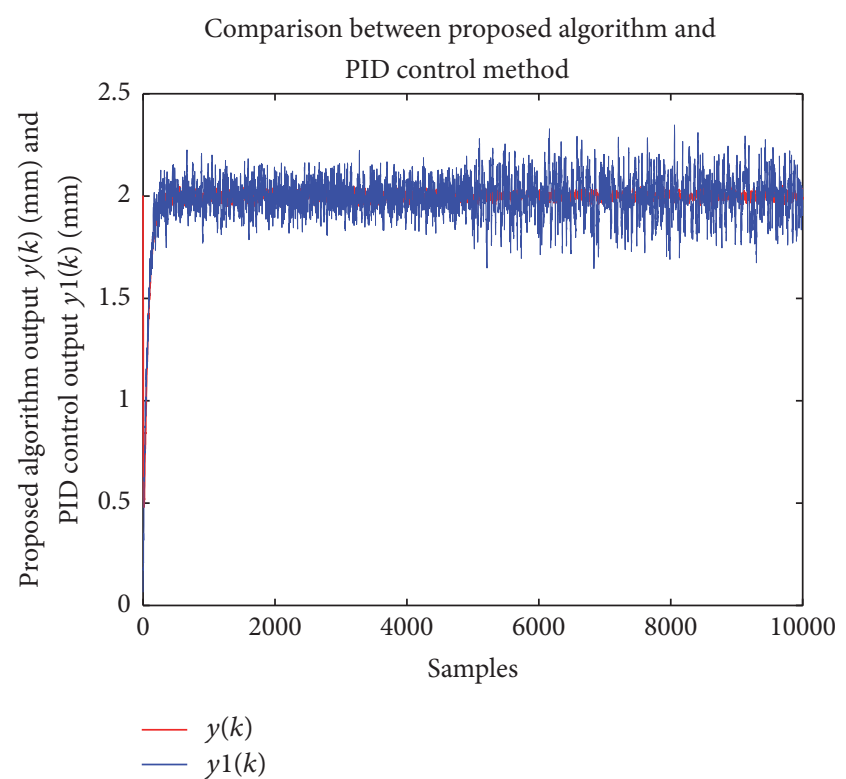

FIGURE 12: Comparison between proposed algorithm (red) and PID control (blue).

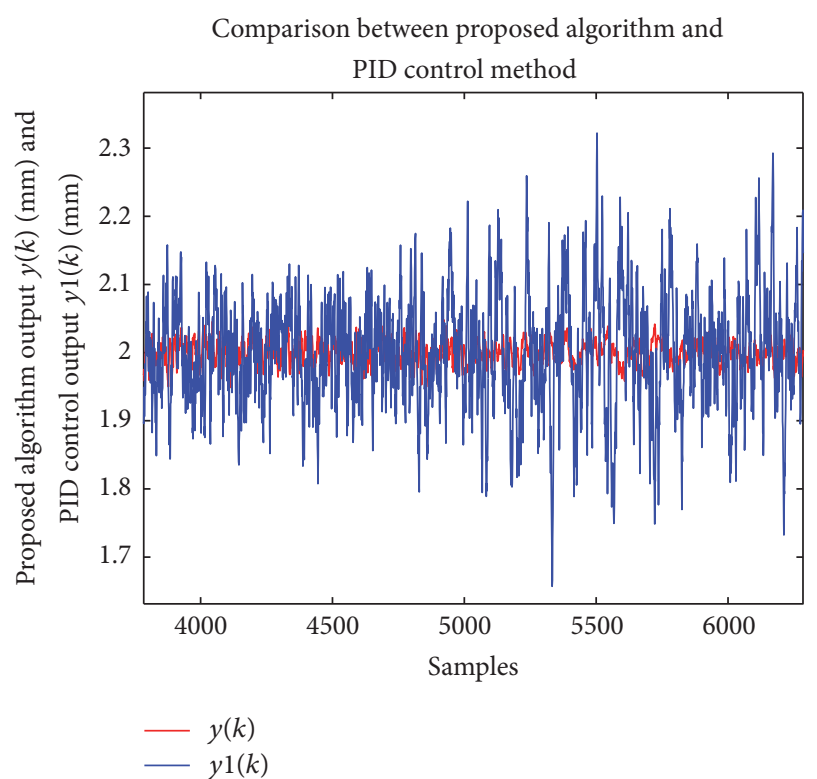

FIGURE 13: Detailed comparison between proposed algorithm (red) and PID control (blue).

Thus, the simulation results show that the proposed approach can effectively monitor the changes of the system parameters and time delay, separate the time delay from the actual data, and feed back the thickness without time delay to the closed-loop, which can greatly reduce the impact of timedelay and parameter uncertainty on the monitoring AGC system. This implies that the proposed system implements better control than the standard PID approach. This improvement can be attributed to the fact that the PID approach cannot take into consideration parameters uncertainty when the system changes, so that the deviations with PID control are larger 
than when using the proposed algorithm, where such factors are taken into account.

\section{Conclusions}

This paper proposes a new identification and control framework for the monitoring AGC system in the rolling process. The framework consists of three steps: time-delay estimation using the cross-correlation function; initial parameter estimation using recursive least squares; and refined parameter estimation using the LM algorithm. The final time delay and model parameters are then used for controlling the process. Simulations based on actual values from a steel rolling mill show that the proposed framework provides better control than the traditional PID control-based approach. Future work will consider examining additional estimation methods in order to determine the best parameters and develop a method to combine fault monitoring with parameter identification and control.

\section{Conflicts of Interest}

The authors declare that they have no conflicts of interest regarding the publication of this paper.

\section{Acknowledgments}

The authors would like to thank the National Natural Science Foundation of China under Grant 61673053, the Beijing Natural Science Foundation under Grant 4162041, and the Fundamental Research Funds for Central Universities under Grant FRF-TP-15-057A3 for funding.

\section{References}

[1] Y. K. Sun, Modelling and control in cold and hot rolling mills, Metallurgical Industry Press, Beijing, 2010.

[2] S. Yin, G. Wang, and H. Gao, "Data-Driven Process Monitoring Based on Modified Orthogonal Projections to Latent Structures," IEEE Transactions on Control Systems Technology, vol. 24, no. 4, pp. 1480-1487, 2016.

[3] M. Dong, C. Liu, and G.-Y. Li, "Robust fault diagnosis based on nonlinear model of hydraulic gauge control system on rolling mill," IEEE Transactions on Control Systems Technology, vol. 18, no. 2, pp. 510-515, 2010.

[4] H. Zhao, N. Lu, and B. Jiang, "Current situation and future trends of fault diagnosis methos for steel rolling process," Steel Rolling, vol. 28, no. 1, pp. 48-53, 2011.

[5] S. Björklund, A Survey and Comparison of Time-Delay Estimation Methods in Linear Systems, Linköping, UniTryck, 2013.

[6] A. B. Rad, W. L. Lo, and K. M. Tsang, "Simultaneous online identification of rational dynamics and time delay: A correlation-based approach," IEEE Transactions on Control Systems Technology, vol. 11, no. 6, pp. 957-959, 2003.

[7] S. Mehrkanoon, Y. A. W. Shardt, J. A. K. Suykens, and S. X. Ding, "Estimating the unknown time delay in chemical processes," Engineering Applications of Artificial Intelligence, vol. 55, pp. 219-230, 2016.

[8] W. Zhong, H. Ge, and F. Qian, "Model identification and control for nonlinear discrete-time system with time delay: a support vector machine approach," in Proceedings of International
Conference on Intelligent Systems and Knowledge Engineering, Chengdu, China, 2007.

[9] X. Kong and V. Solo, "Stochastic averaging analysis of a steepestdescent-type adaptive time-delay estimation algorithm," Mathematics of Control, Signals, and Systems, vol. 7, no. 2, pp. 121-147, 1994.

[10] M. H. Hayes, Statistical Digital Signal Processing and Modeling, John Wiley \& Sons, Inc, New York, USA, 1996.

[11] Z. Liu and C. Li, "Recursive least squares for censored regression," IEEE Transactions on Signal Processing, vol. 65, no. 6, pp. 1565-1579, 2017.

[12] F. Ding, X. Liu, and M. Liu, "The recursive least squares identification algorithm for a class of Wiener nonlinear systems," Journal of the Franklin Institute. Engineering and Applied Mathematics, vol. 353, no. 7, pp. 1518-1526, 2016.

[13] M. Sun, Y. Wang, H. Wang, and Y. Lü, “Time delay control for hydraulic AGC system in cold rolling mill," Hydraulic and Pneumatic, vol. 10, pp. 67-69, 2014.

[14] S. Yin, X. Zhu, J. Qiu, and H. Gao, "State Estimation in Nonlinear System Using Sequential Evolutionary Filter," IEEE Transactions on Industrial Electronics, vol. 63, no. 6, pp. 3786-3794, 2016.

[15] C. Ji, P. Wang, S. Li, and R. Wu, "Neural networks based smith pre-estimated control for time-varrying systems with large time delay," Journal of the Harbin Institute of Technology, vol. 35, no. 3, pp. 303-306, 2003.

[16] X. Wang, J. Chen, and X. Zhu, “The modification and research of identification adaptive predictive control for variable time delay system," Control and Instrument in Chemical Industry, vol. 37, no. 1, pp. 25-27, 2010.

[17] O. J. M. Smith, "Closer control of loops with dead time," Chemical Engineering Progress, vol. 53, no. 5, pp. 217-219, 1957.

[18] X. Xu and Z. Mao, "The neural network predictive control of time-delay systems," Control Theory \& Applications, vol. 18, no. 6, pp. 932-934, 2001.

[19] X. Zhang and S. Xing, "Data-driven predictive control for hydraulic automatic gauge control of hot rolling mills," Control Engineering of China, vol. 22, no. 1, pp. 176-179, 2015.

[20] Y. A. W. Shardt and B. Huang, “Tuning a soft sensor's bias update term. 1. the open-loop case," Industrial and Engineering Chemistry Research, vol. 51, no. 13, pp. 4958-4967, 2012.

[21] Y. A. W. Shardt and B. Huang, "Tuning a soft sensor's bias update term. 2. the closed-loop case," Industrial and Engineering Chemistry Research, vol. 51, no. 13, pp. 4968-4981, 2012.

[22] M. Chen, S. Y. Zhang, and C. N. Tong, "Design of delaydependent robust fault-tolerant $\mathrm{H}_{\infty}$ controller for the strip mill thickness control system," Control and Decision, vol. 26, no. 1, pp. 101-105, 110, 2011.

[23] X. Yang, Q. Zhu, and C. Huang, "Lag stochastic synchronization of chaotic mixed time-delayed neural networks with uncertain parameters or perturbations," Neurocomputing, vol. 74, no. 10, pp. 1617-1625, 2011.

[24] S. Yin, P. Shi, and H. Yang, "Adaptive Fuzzy Control of StrictFeedback Nonlinear Time-Delay Systems With Unmodeled Dynamics," IEEE Transactions on Cybernetics, vol. 46, no. 8, pp. 1926-1938, 2016.

[25] Y. A. W. Shardt, "Statistics for chemical and process engineers: A modern approach," Statistics for Chemical and Process Engineers: A Modern Approach, pp. 1-414, 2015. 
[26] Z.-S. Hou and S.-T. Jin, "A novel data-driven control approach for a class of discrete-time nonlinear systems," IEEE Transactions on Control Systems Technology, vol. 19, no. 6, pp. 1549-1558, 2011.

[27] S. Yin, G. Wang, and H. Karimi, "Data-driven design of robust fault detection system for wind turbines," Mechatronics, vol. 24, no. 4, pp. 298-306, 2014.

[28] X. Yang and C. Tong, "Coupling dynamic model and control of chatter in cold rolling," Journal of Dynamic Systems Measurement \& Control, vol. 134, no. 4, pp. 271-277, 2012.

[29] L. Wang, C. Tong, Q. Li, Y. Yin, Z. Gao, and Q. Zheng, "Practical active disturbance rejection solution for monitoring automatic gauge control system with large time-delay," Control Theory and Applications, vol. 29, no. 3, pp. 368-374, 2012.

[30] L. Ljung, System Identification Theory for the User, Prentice Hall, Inc, Upper Saddle River, New Jersey, USA, 1999.

[31] R. Suzuki, F. Naitou, S. Yoshida, T. Mori, and M. Yamamoto, "Data position detection using fast fourier transform crosscorrelation method for holographic memory," Japanese Journal of Applied Physics, vol. 47, no. 1, pp. 183-188, 2008. 


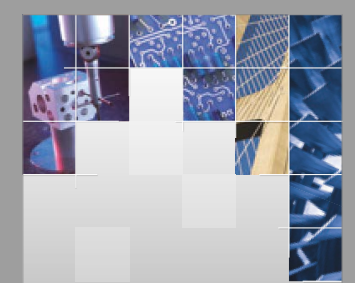

\section{Enfincering}
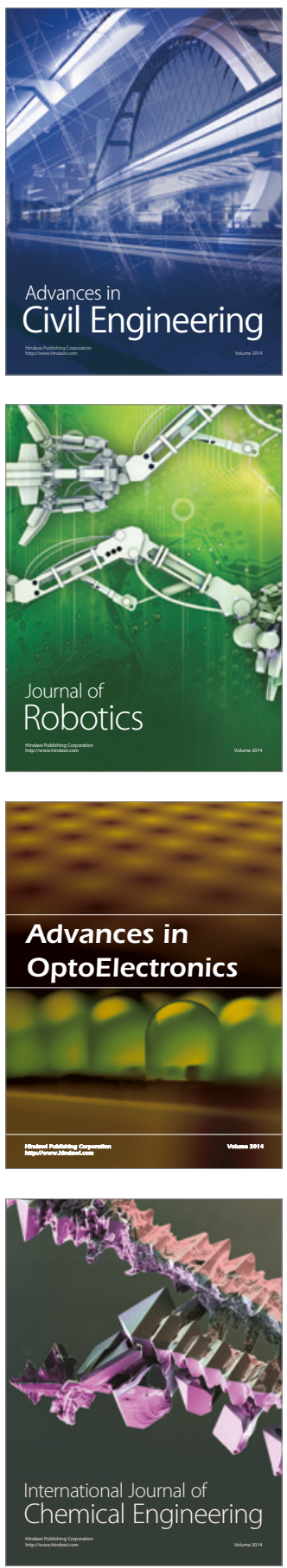

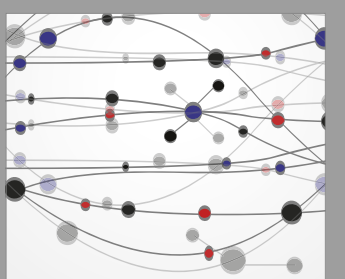

The Scientific World Journal

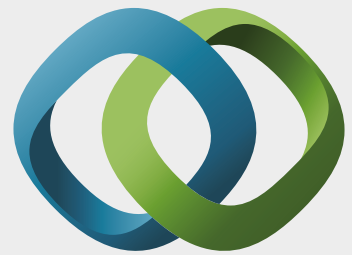

\section{Hindawi}

Submit your manuscripts at

https://www.hindawi.com
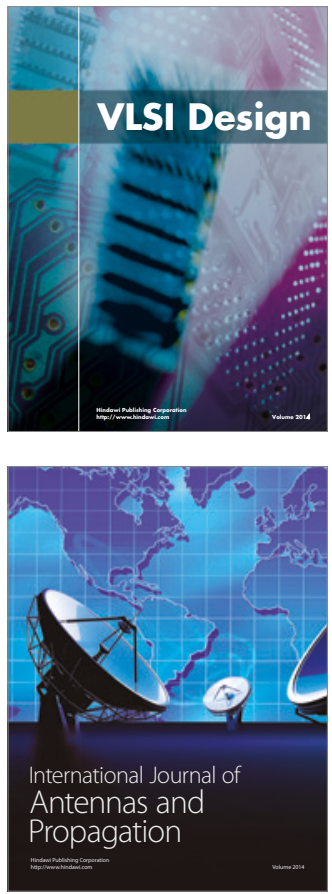

\section{Rotating}

Machinery
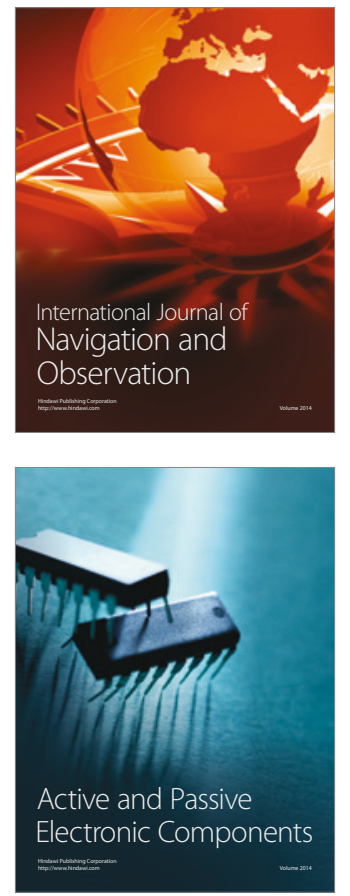
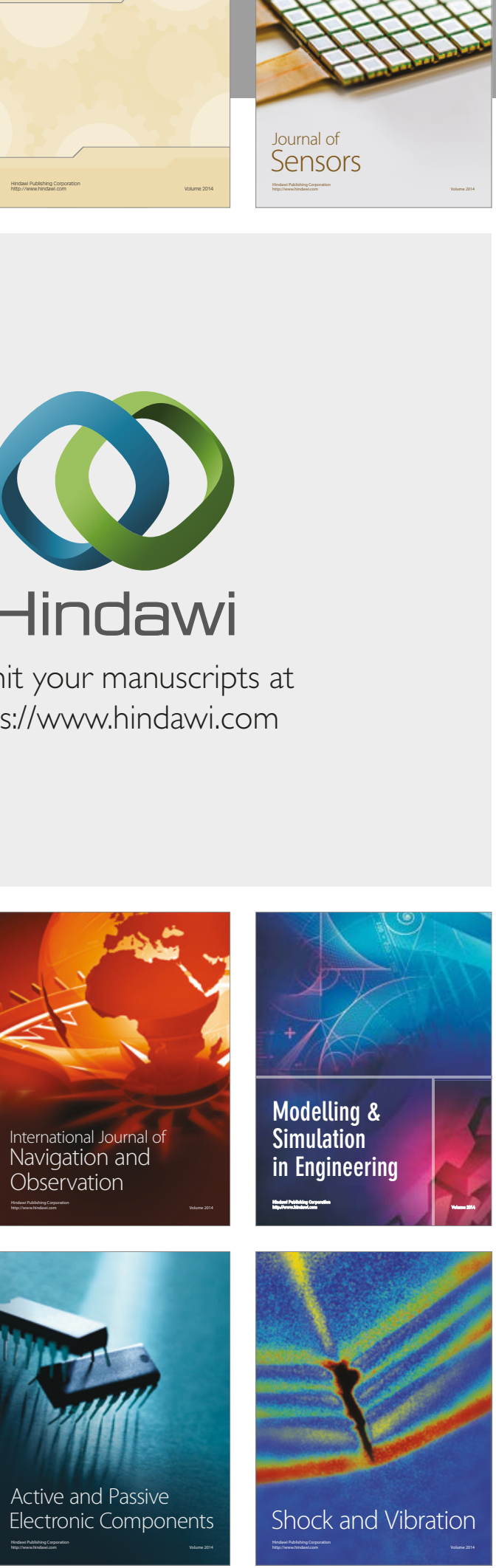
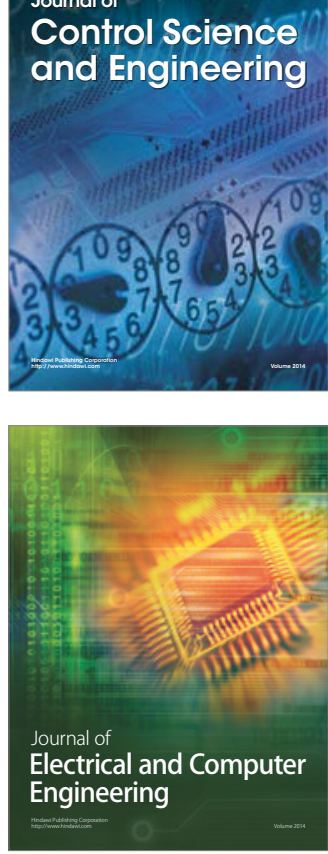

Distributed

Journal of

Control Science

and Engineering
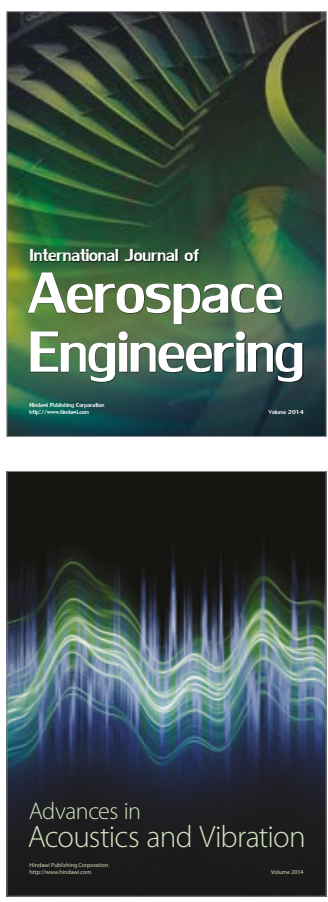

Sensor Networks 